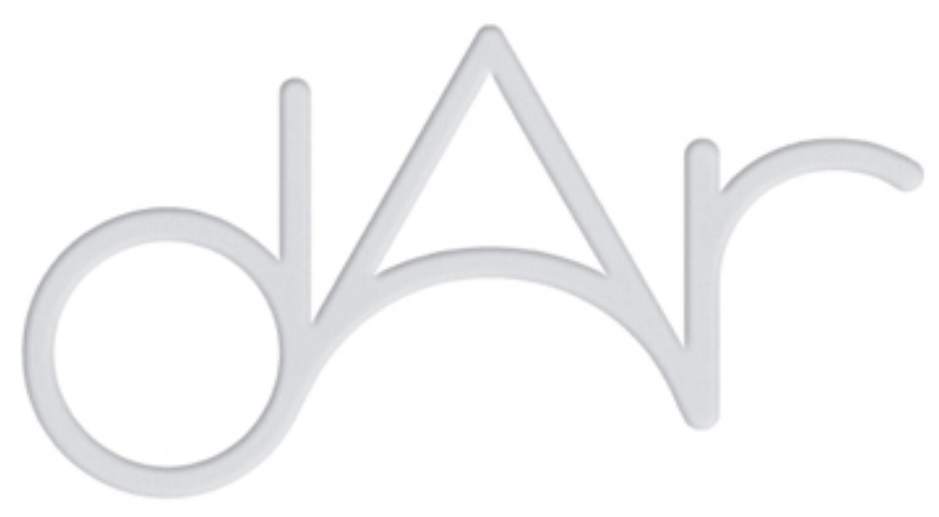

\title{
«Anergui» du Haut Atlas Central: un patrimoine de montagne entre perte de la biodiversité et disparition des valeurs sociétales anciennes
}

Autor(es): $\quad$ Morabet, Rachida El; Ouadrim, M.; Chettar, H.

Publicado por: Imprensa da Universidade de Coimbra

URL

persistente: URI:http://hdl.handle.net/10316.2/44440

DOI: DOI:https://doi.org/10.14195/2182-844X_5_19

Accessed : $\quad$ 26-Apr-2023 06:54:03

A navegação consulta e descarregamento dos títulos inseridos nas Bibliotecas Digitais UC Digitalis, UC Pombalina e UC Impactum, pressupõem a aceitação plena e sem reservas dos Termos e Condições de Uso destas Bibliotecas Digitais, disponíveis em https://digitalis.uc.pt/pt-pt/termos.

Conforme exposto nos referidos Termos e Condições de Uso, o descarregamento de títulos de acesso restrito requer uma licença válida de autorização devendo o utilizador aceder ao(s) documento(s) a partir de um endereço de IP da instituição detentora da supramencionada licença.

Ao utilizador é apenas permitido o descarregamento para uso pessoal, pelo que o emprego do(s) título(s) descarregado(s) para outro fim, designadamente comercial, carece de autorização do respetivo autor ou editor da obra.

Na medida em que todas as obras da UC Digitalis se encontram protegidas pelo Código do Direito de Autor e Direitos Conexos e demais legislação aplicável, toda a cópia, parcial ou total, deste documento, nos casos em que é legalmente admitida, deverá conter ou fazer-se acompanhar por este aviso.

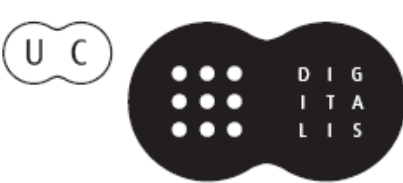



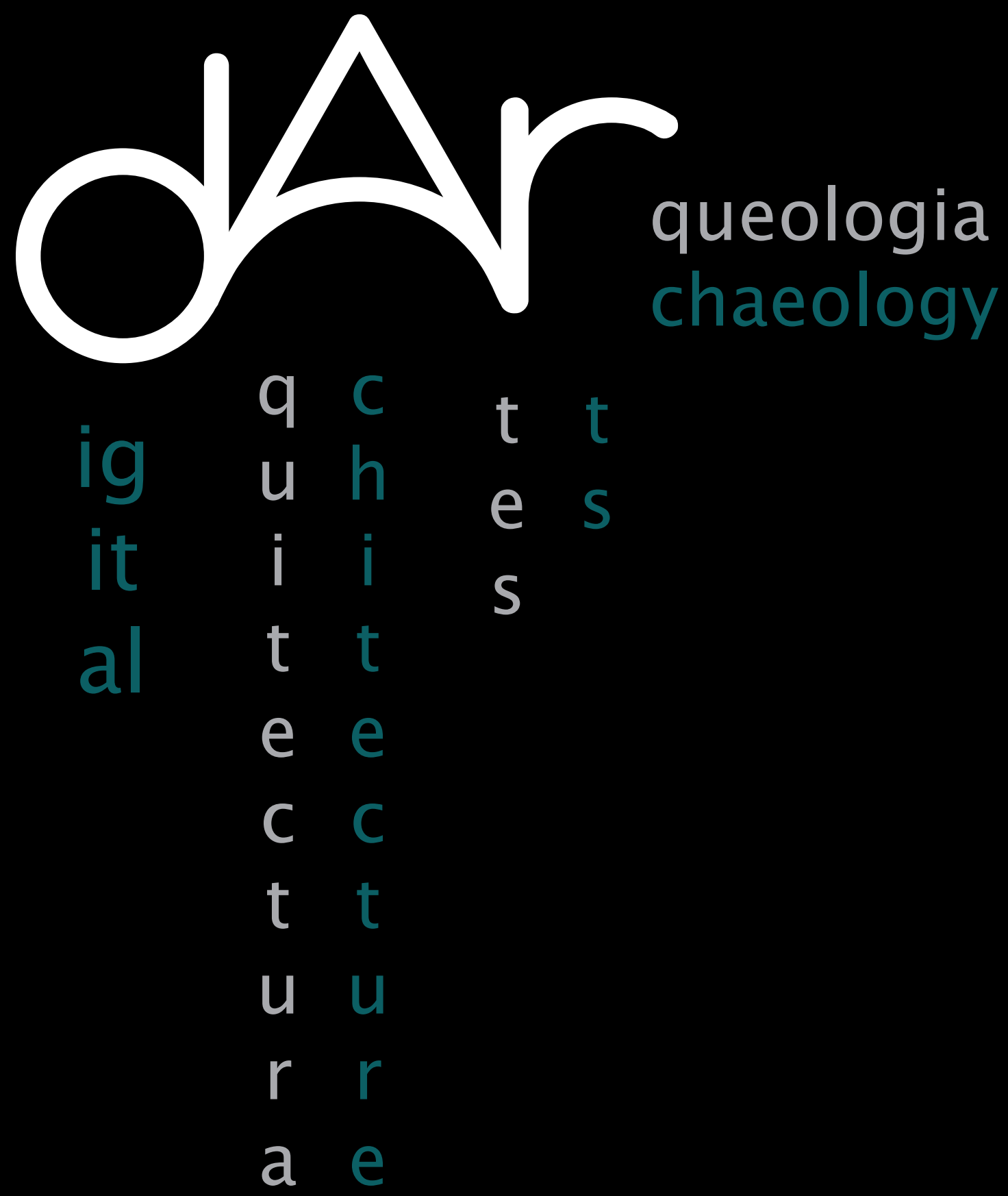

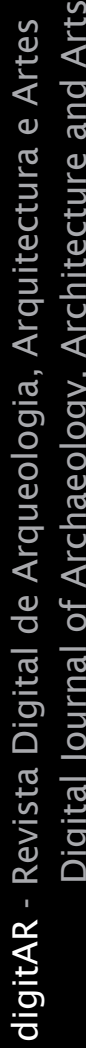


« Anergui » du Haut Atlas Central: un patrimoine de montagne entre perte

DE LA BIODIVERSITÉ ET DISPARITION DES VALEURS SOCIÉTALES ANCIENNES

\author{
Dr. Rachida El Morabet, Dr. M. Ouadrim, H. Chettar \\ FLSH de Mohammedia
}

\title{
Résumé
}

Les montagnes sont dotées d'un patrimoine original et diversifié. En revanche, sur le plan socio-économique, elles restent en retrait par rapport aux bas pays voisins. Dans ce contexte, la valorisation du patrimoine peut être considérée comme une nouvelle chance pour le développement des espaces montagnards. La protection du patrimoine concerne aussi bien le patrimoine naturel que le patrimoine culturel. Le patrimoine géomorphologique et la biodiversité constituent des éléments fondamentaux du cadre de vie des habitants d'un lieu, donc de son environnement au sens strict du terme. L'exemple développé ici concerne les montagnes de «Anergui » du Haut Atlas Central où la forêt et les basses terrasses au bord des fleuves constituent une richesse naturelle et une source de survie de la population locale qui résiste à l'exode rurale.

Préserver et maintenir les connaissances et pratiques des communautés locales, qui incarnent des modes de vie traditionnels, présente un intérêt pour la conservation et l'utilisation durable de la diversité naturelle.

Il ne saurait y avoir de développement soutenable sans la protection de la diversité naturelle comme il ne saurait y avoir de protection de cette diversité sans la préservation du patrimoine culturel des populations autochtones.

Mots-clés: patrimoine, biodiversité, géomorphologie, paysages, identité.

\section{Introduction}

Anergui est un ensemble géographique qui se distingue par son originalité en lien étroit avec ses caractéristiques biophysiques et socioculturelles. Il n'est pas un territoire comme les autres. Par la beauté de ses paysages naturels, il

https://doi.org/10.14195/2182-844X_5_19 
occupe une place particulière. Anergui est aussi un espace singulier où des habitants vivent et travaillent. Un territoire riche de sa culture, sa tradition, son héritage géologique, artisanale, agricole et touristique. Un territoire qui connaît, également, des difficultés liées à l'enclavement, au départ de la jeunesse, etc. Un territoire, dont l'avenir est fragilisé par les changements globaux. Ce basculement s'inscrit dans une dynamique globale que connaît toute la montagne marocaine.

Le système socio-écologique d'Anergui montre une importante transformation. Ce constat est dû à la sévérité des conditions topo-édaphoclimatiques ; sols squelettiques, aridité en conjonction avec la forte pression anthropique exercée sur ces milieux.

Les données exposées dans cet article sont le résultat d'une analyse basée sur l'observation et l'enquête sur le terrain, mais aussi sur une connaissance approfondie de la région, vu qu'un des co-auteurs est originaire d'Anergui et y réside toujours.

\section{Anergui: un espace contraignant en pleine mutation}

Située au Haut Atlas central entre 2456 et 2800 mètres d'altitude, la vallée d'Anergui (province d'Azilal) s'étend sur plus d'une dizaine de kilomètres et abrite une poignée de Douars. Enserrée entre de puissantes chaînes de montagnes culminant à 2860 mètres, cette vallée s'ouvre sur l'extérieur à travers une route goudronnée la reliant à Tagleft distante d'environ $80 \mathrm{~km}$. Cette route est la seule voie de communication de la vallée. Elle permet un certain désenclavement en facilitant tout au long de l'année le transport des hommes et des marchandises.

\section{Aspects morpho écologiques d'Anergui}

La topographie d'Anergui est caractérisée généralement par la variation altitudinale. Anergui est entourée par des crêtes de tous les côtés. Au nord, Par la chaîne de "Tngharf », qui atteint 2546 m, à l'Est, on trouve une série de crêtes de hauteur moyenne, connue localement comme « Asklou n Ozal », dont les altitudes sont de $1495 \mathrm{~m}$, et caractérisée par un important couvert forestier. Vers le sud, nous trouvons la haute chaîne de montagnes Tagounte, où les altitudes dépassent $2786 \mathrm{~m}$. En ce qui concerne la partie ouest de la zone, nous y trouvons la chaîne de montagnes d'Afchtane, dont le point culminant est 2851 mètres. (Fig.1). 


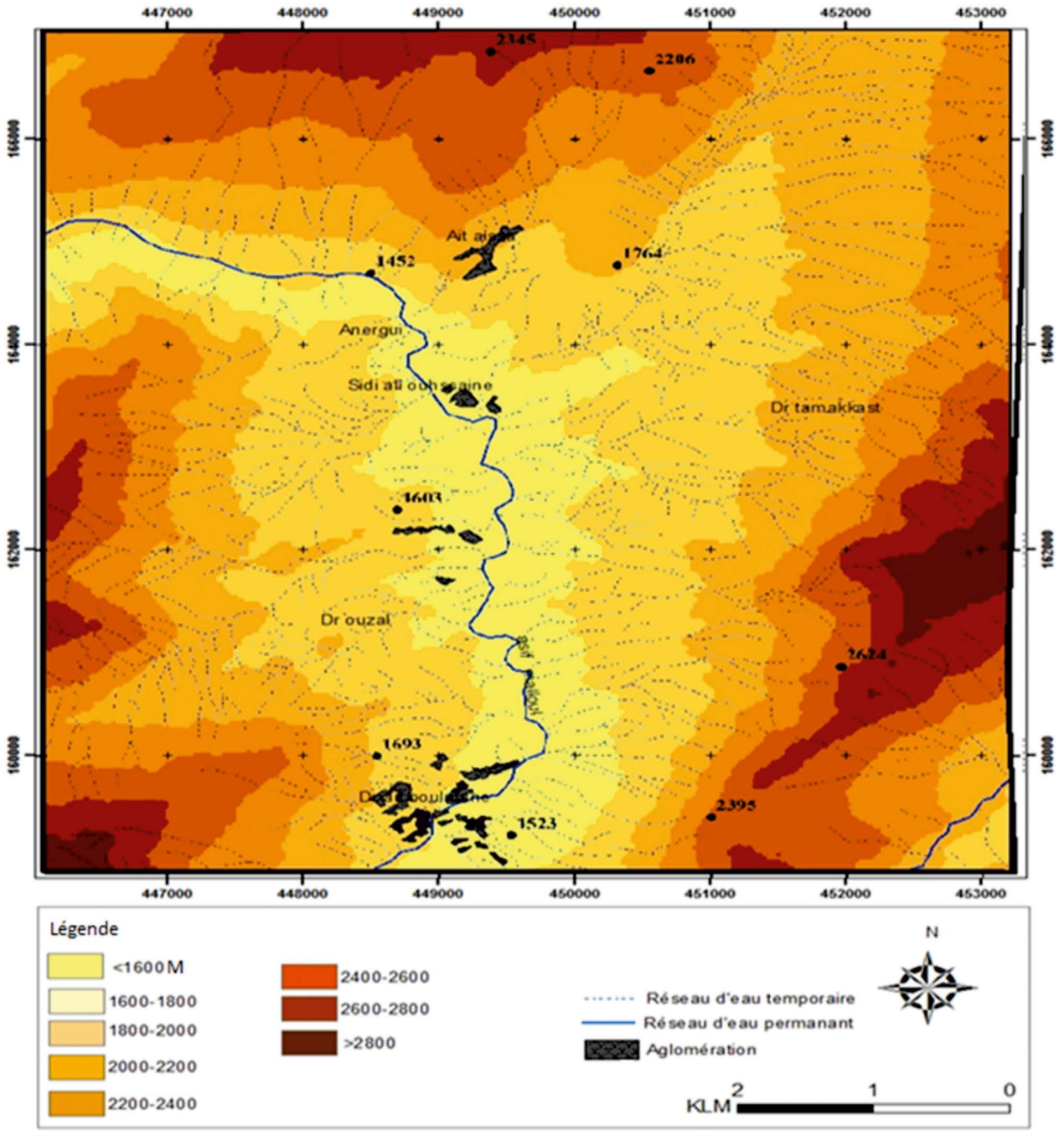

Fig.1. Carte des altitudes d'Anergui

Anergui est traversée par le cours d'eau Assif Melloul, considéré parmi les affluents de oued Ahansal. Ce dernier est un affluent de l'Oued El Abid, qui est un affluent de l'Oued Oum Errabia.

Le climat est de type méditerranéen de par son régime pluviométrique saisonnier, avec des spécificités liées au milieu montagnard. Le régime thermique se caractérise par un été chaud et un hiver froid et parfois très 
rigoureux. L'amplitude thermique est éminente, avec des températures qui varient entre $6^{\circ} \mathrm{C}$ et $+45^{\circ} \mathrm{C}$.

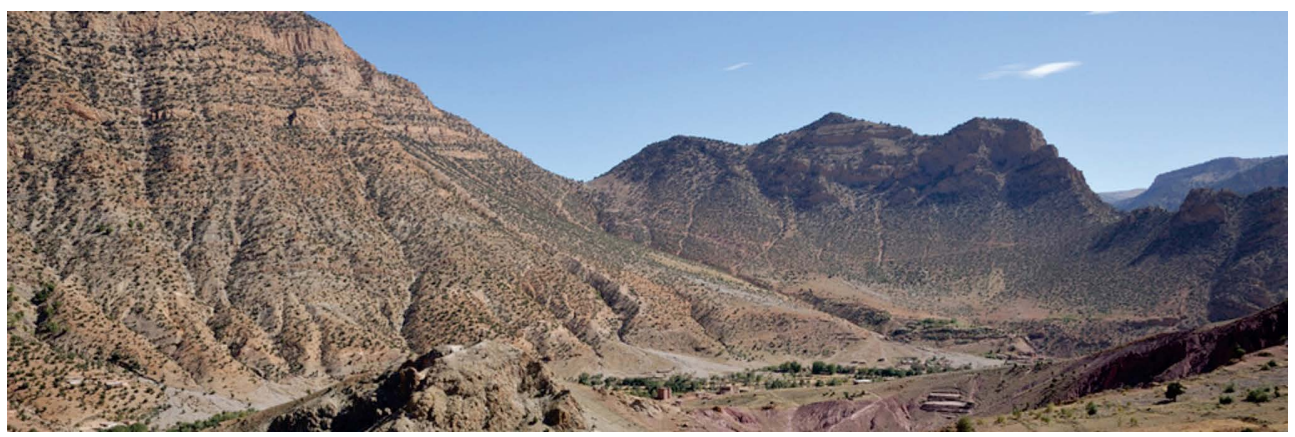

Photo 1 - Vallée d'Anergui (2017). Cliché: Association ANERGUI

Le régime pluviométrique est marqué par des précipitations de saison froide et par un déficit hydrique estival. La forte variabilité des précipitations intra et interannuelles s'accompagne de périodes de sécheresses récurrentes ; il n'est pas rare de trouver des années recevant moins de $300 \mathrm{~mm}$ de précipitations annuelles (Fig.2). Il peut neiger de novembre jusqu'à mai au-dessus de $1500 \mathrm{~m}$.

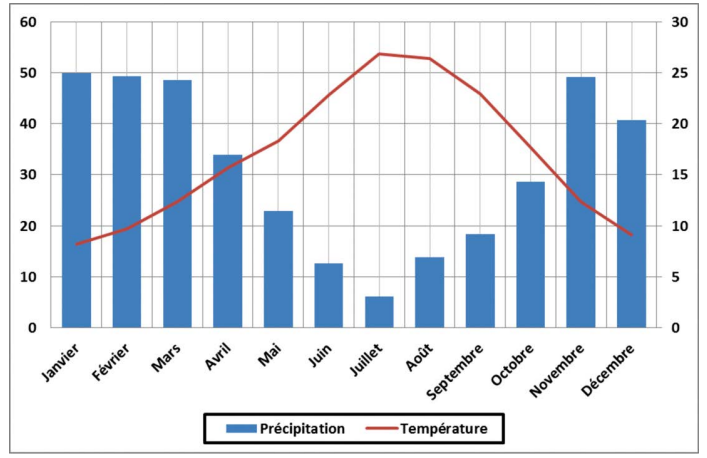

Fig. 2 - La moyenne mensuelle de la température et précipitions entre 1983 et 2013. Station Tellouguit
De par sa position géographique, Anergui reçoit des apports pluviométriques importants qui contribuent à l'alimentation des cours d'eau et des nappes phréatiques. Anergui recèle un réseau hydrographique très diversifié avec un cours d'eau permanantAssifMelloul qui provient de la région d'Aghbala. En ce qui concerne les cours d'eau saisonniers, ils sont répartis le long des pentes et vallons.

Anergui illustre le principe de verticalité de Garrigues-Cresswell (1987). Les bioclimats de Anergui vont du semi-aride supérieur dans le fond de la vallée au subhumide sur les versants les plus arrosés.

Trois étages écologiques se superposent le long du gradient d'altitude :

- Le fond de vallée entièrement aménagé en périmètre irrigué cultivé ; 
- Entre 1700 et 2500 m d'altitude, on trouve l'espace boisé plus ou moins défriché pour l'installation de cultures vivrières bour ;

- Les zones d'altitude utilisées comme parcours.

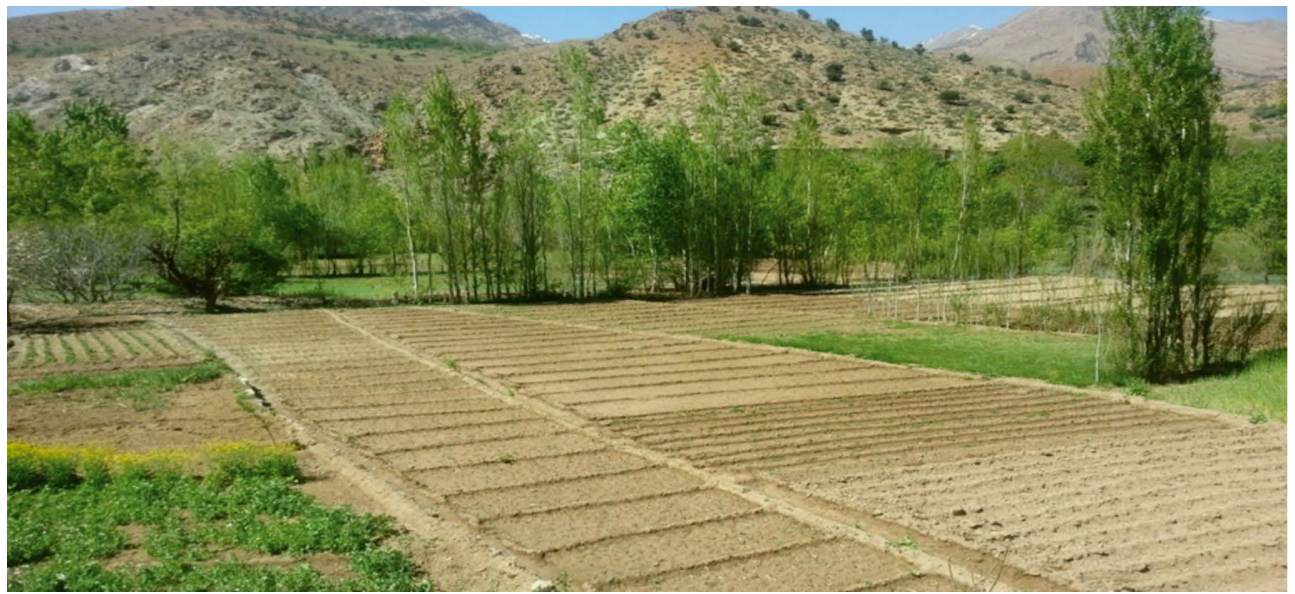

Photo. 2 - Le fond de vallée entièrement aménagé en périmètre irrigué cultivé. (Terrain, 2017)

Les terres agricoles irriguées sont de statut privé (Melk). Les espaces sylvopastoraux sont collectifs et leur accès est en général réservé aux membres de la communauté villageoise (Taqbilte); ils sont soumis à des règles très strictes (Tgdalt).

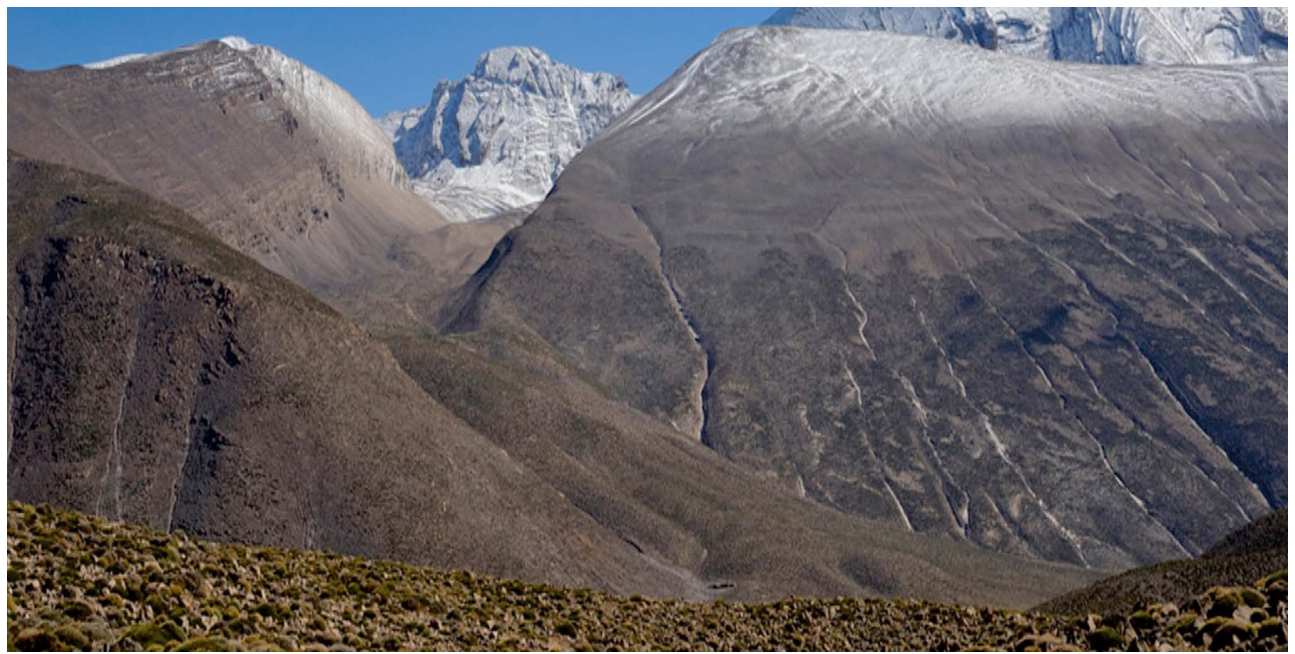

Photo. 3 - Les crêtes d'Anergui (2017). Cliché : Association ANERGUI 


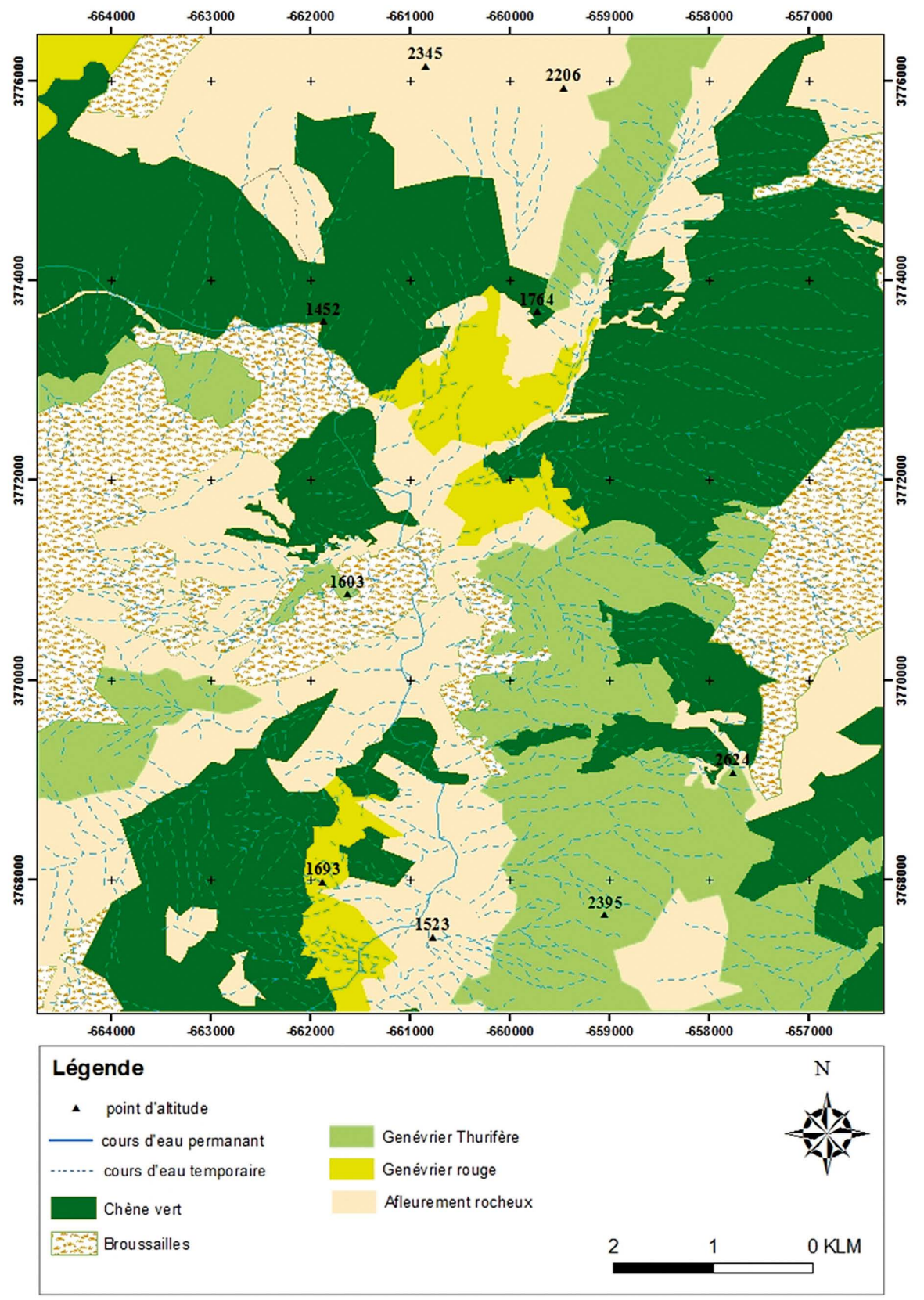

Fig. 3 - Couvert végétal d'Anergui selon les eaux et forêts (2016) 
Les systèmes de production d'Anergui reposent sur l'exploitation accommodée de ces trois étages écologiques, assemblant l'agriculture irriguée et des activités d'élevage qui éprouvent d'importants changements. Les espaces forestiers à usages variés jouent également un rôle essentiel pour les villageois : fourrage foliaire, bois d'œuvre et de chauffage, plantes aromatiques et médicinales etc.L'espace forestier est compartimenté en différentes zones (Fig.3):

La végétation de la région reflète bien l'étagement bioclimatique méditerranéen,quant aux paysages végétaux, ils sont fortement façonnés par les pratiques agropastorales et la gestion forestière traditionnelle.

\section{Une couverture végétale fortement dégradée / Une forte érosion des sols}

Anergui est un espace fragile caractérisé par des formations végétales de type matorral dominant, souvent très ouvertes et protégeant mal les sols pauvres, peu évolués et peu profonds.

L'étude des images satellitaire (Landsat) des années 1984 \& 2015 (Fig.4) donne uneidée sur l'évolution de la végétation : un appauvrissement frappant pour les terres agricoles et pour les plantations.

- En 1984, la végétation représentait 34.08 \% de la superficie totale de la zone étudiée.

- $\quad$ En 2015, cette proportion s'est vue diminuée à 22,05 \% .

Dans le contexte climatique à précipitations violentes, notamment en automne après le dessèchement intense des mois estivaux, l'érosion par ravinement et décapage aréolaire est très intense.

Cette dégradation est également liée aux fortes pressions anthropiques exercées sur les milieux. Malgré les relatives faibles densités de population, la fragilité globale de ces terres marginales en démultiplie les effets. Le surpâturage pèse également lourdement sur les écosystèmes déjà fragilisés. Le système agro-sylvo-pastoral crée une forte charge sur l'écosystème.

La cruauté des processus d'érosion dans cette région se lit également à travers les taux d'envasement des barrages. L'érosion spécifique moyenne était estimée à $240 \mathrm{~m} 3 / \mathrm{km}^{2} /$ an dans les années 1990 . Elle se traduit par l'envasement du lac du barragede Bin el Ouidane, estimé à $1.50106 \mathrm{~m} 3 / \mathrm{an}$, qui a fait perdre $183.7 \mathrm{Mm} 3$ de capacité de rétention d'eau, soit $12.4 \%$ du volume initial qui était de $1484 \mathrm{Mm} 3$ (soit une perte d'environ $4.5 \mathrm{Mm} 3$ par an). 


\section{Patrimoine d'Anergui: une richesse naturelle et culturelle}

Anergui héberge une variété de géomorphosites, de paysages spectaculaires et de haute valeur patrimoniale. En raison de sa situation au cœur de la chaîne atlasique, ce territoire se caractérise par des structures géologiques très spécifiques qui marquent le paysage : dolines, combes, cluses, lapiés, canyons, résurgences et vallées sèches.

C'est grâce à cette richesse qu'une partie de la région a été labellisée en Géo Parc M'Goun par l'UNESCO, en septembre 2014.

En outre, la richesse du territoire étudié ne se limite pas à son patrimoine naturel, mais également à son patrimoine culturel, riche et diversifié, que l'on peut résumer comme suit :

$\checkmark \quad$ Le patrimoine culturel matériel, notamment architectural : (zaouiat, ksour et kasbahs, marabouts, greniers, citadelles, ). En effet, l'architecture impressionnante, qui jaillit au cœur de ces paysages grandioses et inaccessibles, a d'abord eu une vocation défensive et militaire. Un certain nombre de ces bâtisses sont heureusement sous la protection de l'UNESCO. Le patrimoine culturel riche et diversifié est en général reconnu mais peu valorisé, voire même laissé à l'abondant.

$\checkmark \quad$ Le patrimoine culturel immatériel:(coutumes, costumes traditionnels, moussems ...). Le patrimoine immatériel est également riche dans cette région, appuyé notamment sur une tradition orale ancestrale.
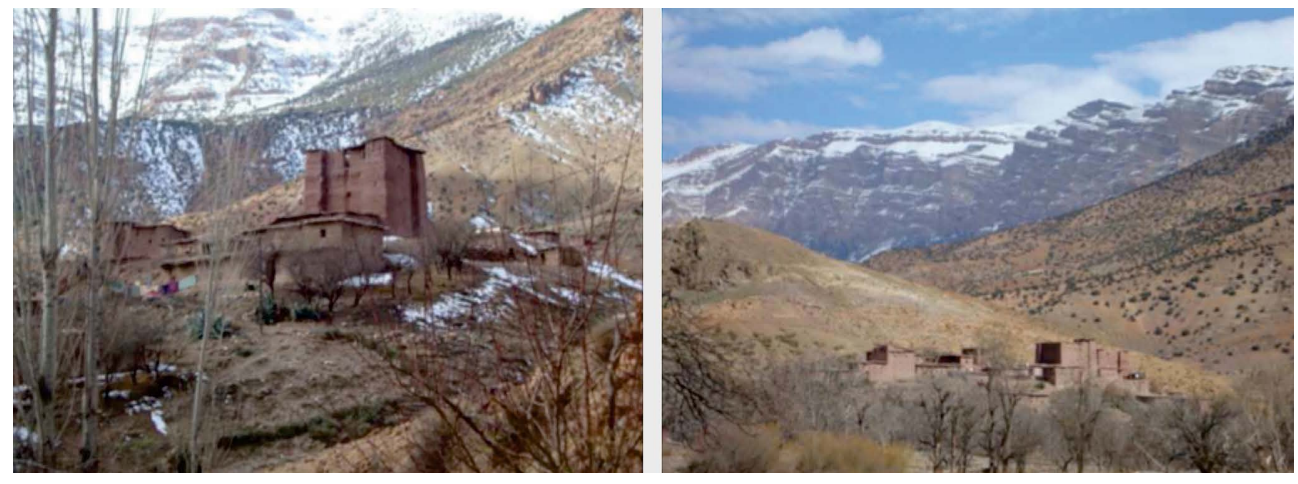

Photo. 4 - Greniers citadelles, Anergui (2013). Cliché : Moulay Askour

Le patrimoine ainsi mis en lumière doit servir au développement économique d'Anergui, dans une perspective de développement soutenable, par le biais 
de l'éco-géo-tourisme par exemple.

Ce qui menace aujourd'hui cette culture millénaire est perfide : l'exode rural, le chômage, la sécheresse, qui d'année en année se fait plus agressive, causée par des changements planétaires venus de très loin.

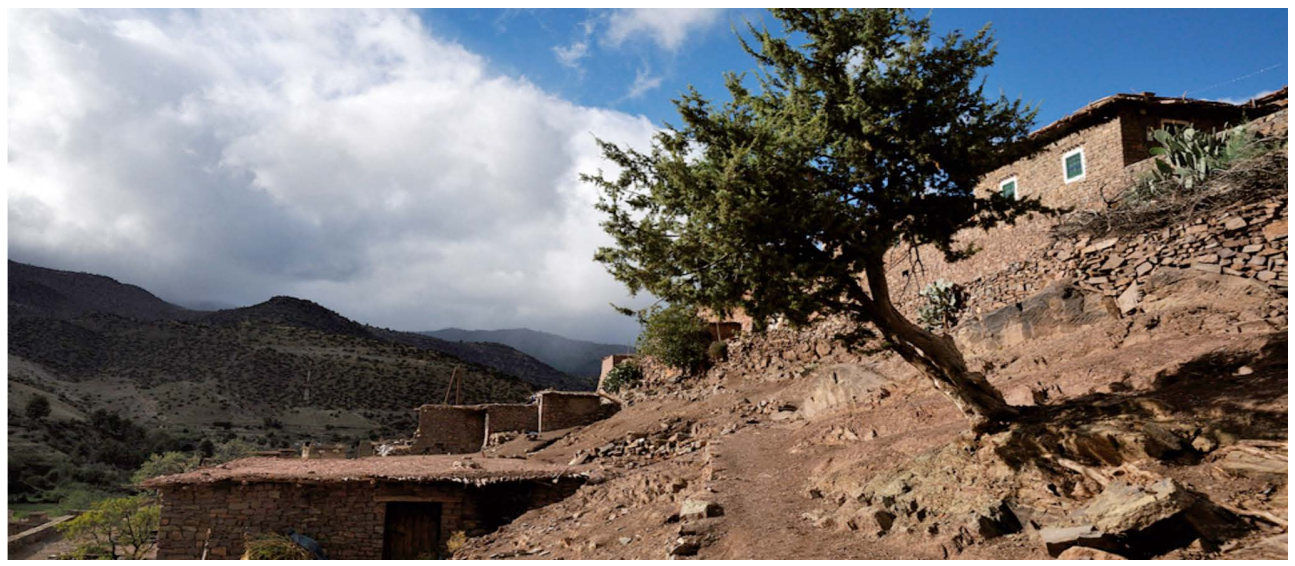

Photo 5 - Village Anergui, 2017. Cliché: Association ANERGUI

\section{Le développement socioéconomique d'Anergui.}

L'agriculture et l'élevage constituent les principales activités d'Anergui. La surface agricole utile est très faible, les sols sont pauvres, les cultures se concentrent dans le fond de la vallée où elles sont pratiquement exclusivement irriguées par dérivation des eaux des oueds.

Le système agro-sylvopastoral où les cultures saisonnières de céréales, légumes et fruits sont complétées par l'élevage (viande, lait, animaux de trait) est uniquement vivier. La forêt fournit combustible, bois d'œuvre et une partie du fourrage.

Les moyens utilisés pour les travaux de labourage des terres sont rudimentaires et non mécanisés où $75 \%$ des superficies sont travaillées à l'aide de charrues en bois contre $1 \%$ seulement par des araires.

La taille des parcelles cultivées est globalement faible où $80 \%$ des exploitations ont moins de 5 ha et $16 \%$ une taille de 5 ha à moins de 10 ha.

La céréaliculture est la principale activité agricole où la culture de l'orge couvre quasiment toutes les terres en montagne. Le blé dur vient en seconde place, suivie par la culture du blé tendre.

L'élevage constitue une des activités majeures d'Anergui, où les contraintes climatiques, topographiques et pédologiques et la pauvreté des populations la dédie essentiellement à un élevage caprin qui y est dominant avec l'élevage ovin. 


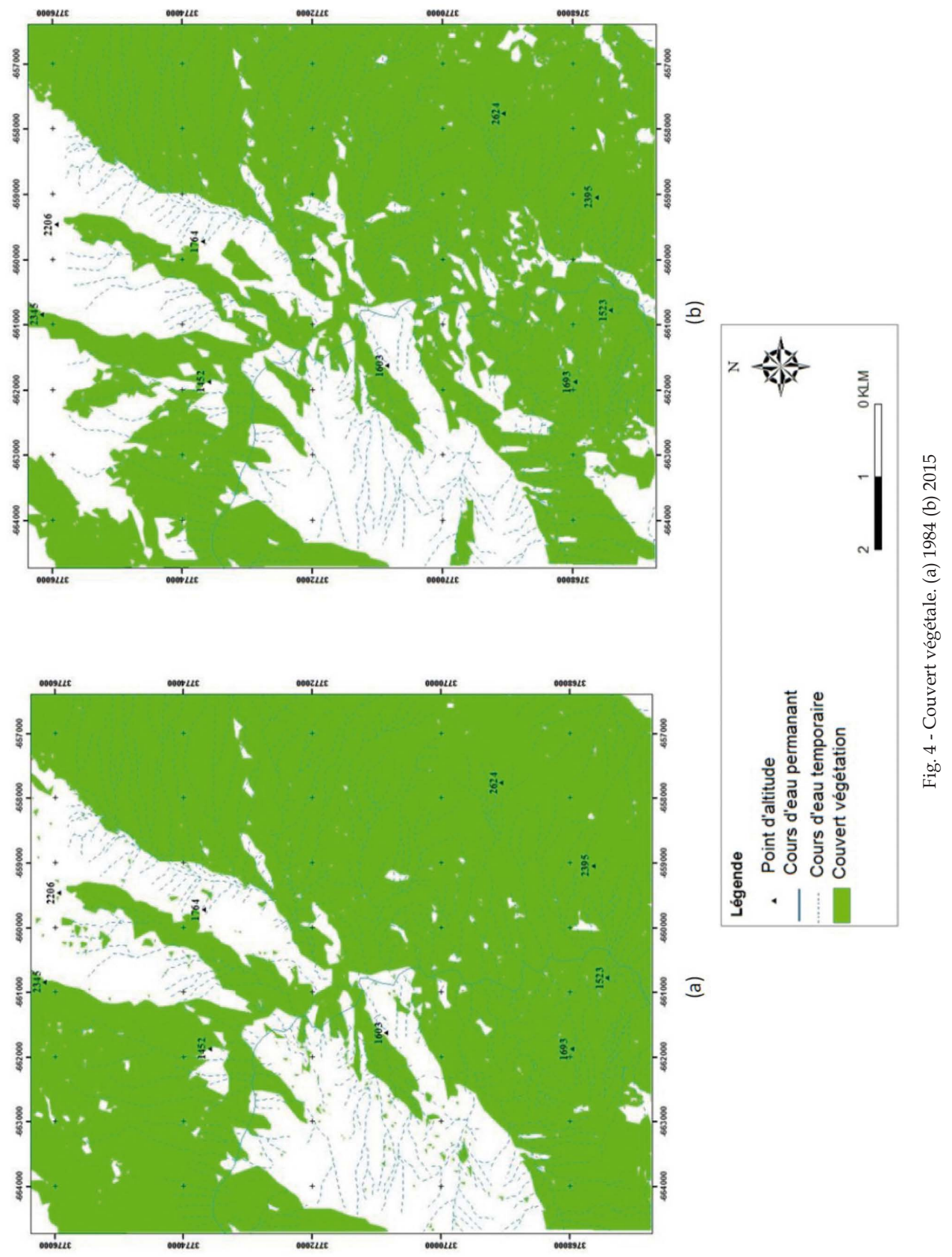


Les autres types d'élevage se partagent entre le mulet en montagne pour les déplacements sur les sentiers, les ânes dominants dans les zones moins abruptes, et le cheval réservé essentiellement à la magnificence et les fantasias. Il est édifiant de constater à quel point dans Anergui, un effort a été consenti dans le but d'harmoniser la vie traditionnelle avec des pratiques touristiques, créations de refuges, de gîtes tout en cherchant à préserver l'authenticité de l'accueil Amazigh (Des pratiques éco-touristiques associées aux gites et au trekking).

S'ajoutent au développement des activités liées au tourisme, les interventions croissantes de l'État, des institutions de développement et des associations locales : création d'une école de guides et de gîtes d'étape, raccordement récent au réseau national d'électricité et de téléphonie mobile, construction d'une route, réalisation de projets de développement, etc.

Toutefois, il faut constater queles temps changent et d'envisager le développement en misant principalement sur la sagesse locale en matière de changement en douceur.

Le contexte socio environnementale de la région est à l'origine de différents risques plus ou moins identifiés.

Leurs impacts et leur ampleur sont fonction des caractéristiques physiques, biophysiques et des modes d'occupation développés dans la région (Fig.5).

On distingue les risques qui concernent la dégradation des ressources naturelles ; les sols et le couvert végétal font partie des risques identifiés à l'échelle de toute la région. Si les processus biophysiques et hydrologiques qui génèrent certains processus comme l'érosion des sols sont bien connus, le rôle et les impacts d'autres facteurs comme l'agriculture ou l'élevage sur l'évolution du couvert végétal forestier par exemple, constituent encore un sujet d'étude et de débat. En effet, il est important d'inscrire ces phénomènes d'évolution dans une dynamique globale des paysages de la région, et de nuancer les discours alarmistes sur la dégradation de ce patrimoine.

L'enclavement de la montagne et les faibles opportunités d'emplois en dehors de l'agriculture familiale avec de forts taux de chômage, favorisent un fort exode rural des populations jeunes.

\section{Conclusion}

L'ensemble des transformations que connaît Anergui se sont traduites par différents enjeux. Ils sont à la fois de nature écologique, sociale et culturelle. Si les conséquences de certains sont déjà visibles, leurs impacts sont difficilement mesurables. 


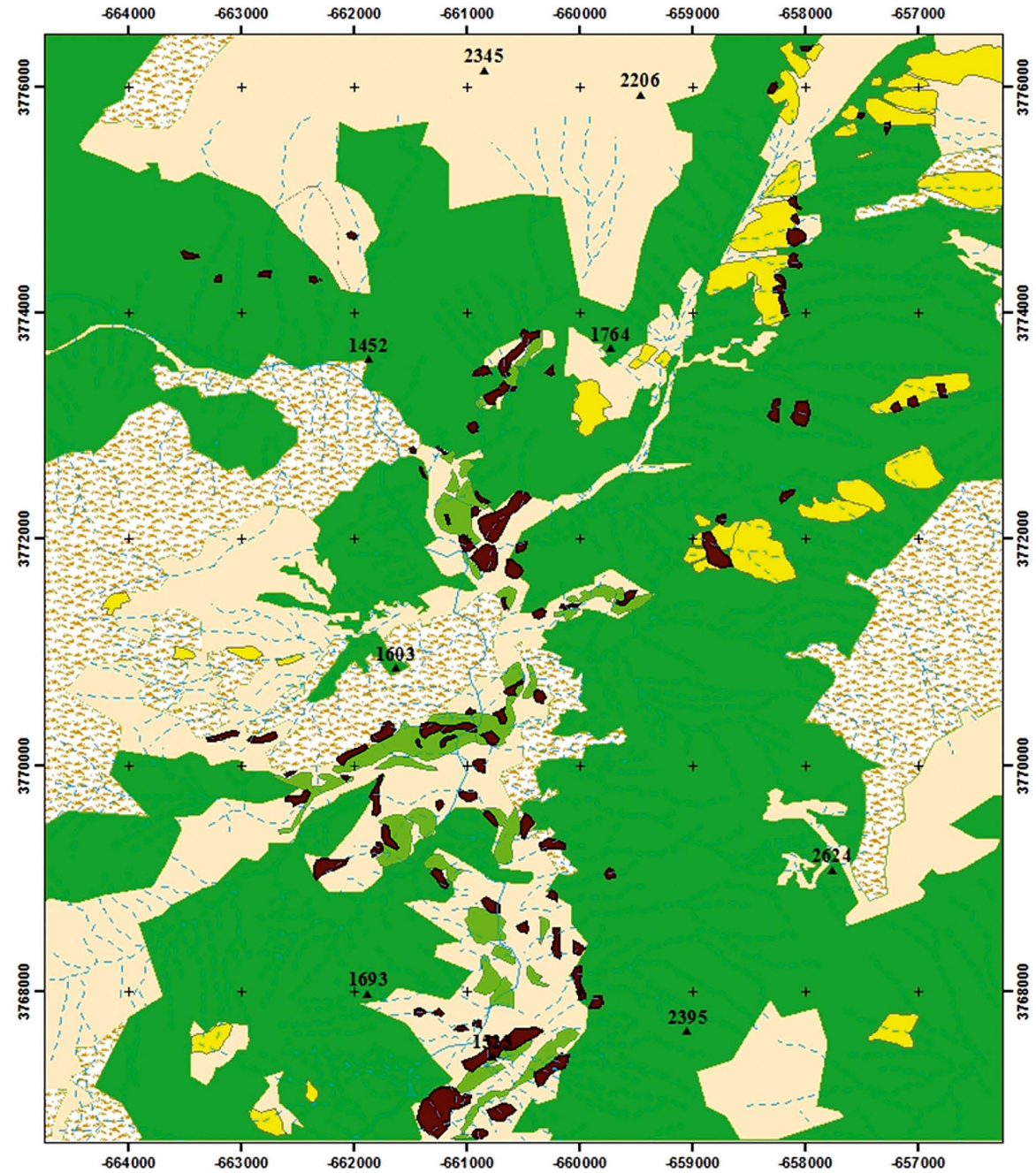

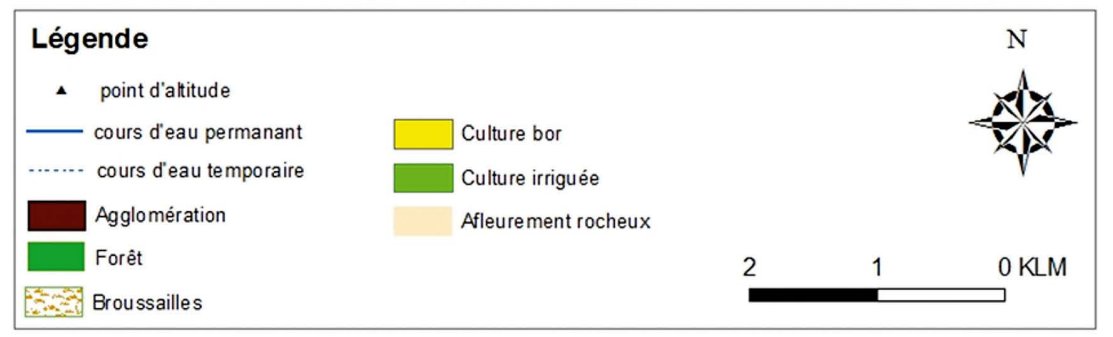

Fig. 5 - Occupation du sol 
Le discours alarmiste de la pression sur les systèmes écologiques et paysagers de la montagne ; notamment d'Anergui ; est d'actualité. Ces discours s'appuient sur une lecture scientifique schématique basée sur des analyses sans prérequis historiques de la dynamique des paysages de la montagne marocaine. Ces analyses sont à nuancer,vu que les dynamiques ne s'ordonnent pas, elles s'appuient sur une profondeur culturelle et naturelle. Toute action économique ou socio-culturelle pour le développement, doit être une opportunité pour associer les populations à un processus d'évolution de leurs perceptions de leurs paysages, de leur patrimoine et de leur cadre de vie.

\section{Bibliographie}

2010 زويللوي - ي

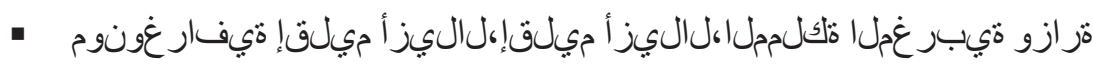
2010

- Agence du bassin versant Oum rabia.2015

- Association Anergui : http://www.anergui.org/

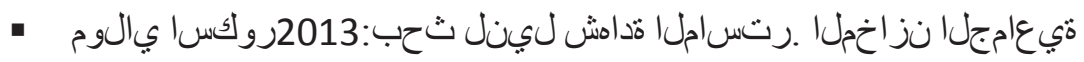

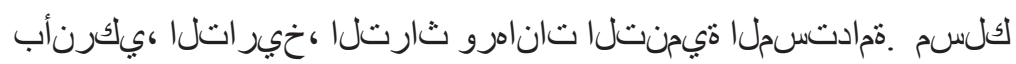

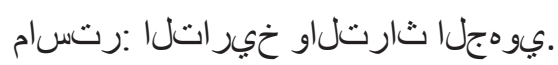

